\title{
Le peuplement simulidien de la Tunisie : I. Inventaire faunistique et biogéographie (Diptera-Nematocera)
}

\author{
M. Boumaiza 1 \\ M. Clergue-Gazeau ${ }^{2}$
}

Mots-Clés : Simuliidae, Diptera, Tunisie, faunistique, zoogéographie.

Dans ce premier travail, les auteurs donnent une liste des Simuliidae récoltés dans les principaux réseaux hydrographiques tunisiens. Neuf espèces y sont citées pour la première fois. L'étude biogéographique prouve qu'il s'agit principalement d'une faune paléarctique à distribution ouest ou circum-méditerranéenne. Les éléments d'origine éthiopienne ou orientale sont faiblement representés.

The Simulildae of Tunista : I. Faunistic list and zoogeography.

Keywords : Simuliidae, Diptera, Tunisia, faunistic, zoogeography.

In this first study, the authors list the Simuliidae collected in the main tunisian hydrographic networks. Nine species are cited for the first time. The biogeographical analysis shows that we are essentially dealing with a palearctic fauna with a west or circum-mediterranean distribution. Ethiopian and oriental elements are poorly represented.

\section{1. - Introduction}

Fort peu connue, la faune simulidienne de la Tunisie présente un intérêt évident à cause de la situation géographique de ce pays, à l'ouest du bassin méditerranéen, entre le sud-ouest de l'Europe et la région éthiopienne. C'est la raison pour laquelle nous avons entrepris la prospection d'une grande partie des réseaux hydrographiques de ce pays, en partant des petites rivières de la côte Nord et en descendant vers le Sud jusqu'aux confins des oasis sahariens.

Cette note expose les résultats d'un premier travail entrepris sur une telle étendue du territoire : auparavant, certains auteurs avaient décrit (Puri, 1925) ou cité (Colas-Belcour, 1931) l'espèce Simulium (Wilhelmia) pseudequinum Séguy, capturée au cours de prélèvements ponctuels; de même pour Simulium (Simulium) reptans (L.) (Anderson et Lehucher, 1940) dont la présence est d'ailleurs actuellement contestée. La première et unique campagne

1. Laboratoire d'Écologie et de Biologie animale, Département des Sciences Biologiques. Faculté des Sciences de Tunis, Tunisie.

2. Université Paul Sabatier, Laboratoire d'Hydrobiologie, UA 695 du C.N.R.S., 118, route de Narbonne, 31062 Toulouse Cédex, France. de récoltes sur ce groupe fũt celle de BaillyChoumara et al. (1970) : elle couvre les cours d'eau septentrionaux de l'est à l'ouest du pays. Par conséquent, des recherches sur ces Invertébrés s'imposaient dans les réseaux hydrographiques de l'axe nord-sud : elles nous ont permis d'obtenir de nouvelles données faunistiques et biogéographiques.

Par ailleurs, cette famille a fait l'objet d'un certain nombre de travaux récents et anciens sur les territoires algérien et surtout marocain ; il était donc urgent d'acquérir des connaissances sur les Simulies de l'est du Maghreb.

Une note ultérieure traitera de la biologie et de l'écologie des espèces citées.

\section{2. - Méthodes et localités d'étude}

Composé de larves (L), de nymphes (N) et d'exuvies nymphales $(E)$, le matériel a été récolté à partir de prélèvements benthiques par l'un de nous (Boumaiza) à l'aide d'un filet Surber (vide de maille $0,3 \mathrm{~mm}$ ) pour les mesures quantitatives, et de dérive (vide de maille $0,2 \mathrm{~mm}$ ). La fixation des animaux se fait au formol à $5 \%$, la conservation dans l'alcool à $70 \%$. Quant au nombre d'imagos capturés au filet 
entomologique, il est restreint et nous n'avons pu en tenir compte dans ces premiers résultats.

Une étude physico-chimique des 36 stations prospectées est achevée (Boumaiza 1984) : elle présente les principaux paramètres des réseaux hydrographiques étudiés et l'on peut s'y rapporter pour plus de détails sur le résultat des diverses mesures.

Les campagnes de récoltes se sont échelonnées sur quatre années : novembre 1980 , de février à novem. bre 1982, d'avril à novembre 1983, février et juillet 1984 et avril 1985. Dans la mesure du possible, nous avons essayé de prospecter le plus grand nombre de stations à une même époque de l'année.

Les 36 localités prospectées sont consignées sur une carte de la Tunisie (fig. 1) et sur le tableau I résumant les principaux paramètres utilisés dans ce travail : les stations 1 à 17 sont situées dans le bassin de la Medjerda, 18 à 23 dans les cours d'eau du nordouest, 24 à 27 dans le réseau du lac Ichkeul, 28 à 32 à l'est, 33 à 36 dans les réseaux méridionaux.

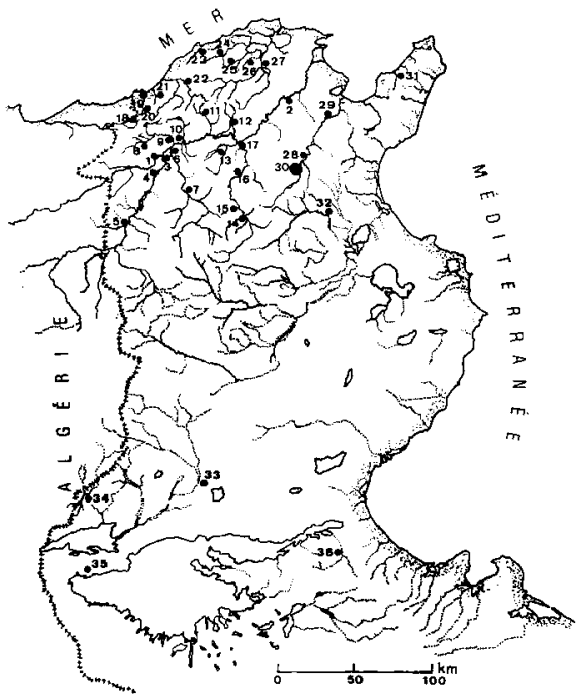

Fig. 1. Localisation des stations d'étude et répartition de $S$. (W.) pseudequinum : - petits cercles noirs : répartition de $S$. (W.) pseudequinum - petits cercles noirs + grand cercle noir : stations d'étude.

\section{3. - Faunistique}

Nous donnons ci-dessous dans l'ordre de la classification de Crosskey (1981), la liste des quatorze espèces répertoriées; le nombre de localités étant important, nous les avons rassemblées sur 3 cartes dont les numéros sont indiqués après chaque nom d'espèce.

\section{Prosimuliini}

** Prosimulium (Prosimulium) groupe rufipes

$\mathrm{N}$ (fig. 2)

** P. (Urosimulium) juccii Contini, 1966

* Metacnephia blanci Grenier et Theodorides, 1953

N, E (fig. 2)

L, N, E (fig. 3)

Simuliini

Simulium (Eusimulium) latinum

Rubzov, 1962

L, N, E (fig. 3)

* Simulium (E.) groupe aureum

S. (Nevermannia) cryophilum

Rubzov, 1959

N, $E$ (fig. 2)

* * S. (N.) ibleum Rivos, 1966

L, N, E (fig. 2)

$S$. $(N$.) groupe angustitarse

L (fig. 2)

N, E (fig. 3)

* $S$. (N.) ruficorne Macquart, 1835 L, N, E (fig. 3) S. (Odagmia) nitidifrons Edwards, 1920

* $S$. (O.) ornatum Meigen, 1818

** S. (Simulium) argenteostriatum Strobl, 1898

L, N, E (fig. 2)

L, N, E (fig. 2)

S. (Wilhelmia) pseudequinum

Séguy, 1921

L, N, E (fig. 2)

* $S$. (W.) sergenti Edwards, 1923

L, N, E (fig. 1)

L, N, E (fig. 2)

** Especes nouvelles pour l'Afrique du Nord.

* Espèces nouvelles pour la Tunisie.

\section{Répartition du peuplement en Tuniste}

Bailly-Choumara \& al. (1970) ont dressé une liste de 8 taxa récoltés dans plusieurs ruisseaux de la région septentrionale du pays: $S$. (Boophthora)? erythrocephalum, de Geer, S. (E.) latinum, S. (Hellichiella) yerburii Edwards, $S$. (N.) cryophilum, $S$. (N.) vernum Macquart, $S$. (N.) sp., $S$. (O.) nitidifrons et $S$. (W.) pseudequinum. Quatre espèces se retrouvent dans nos prélèvements : $S$. (W.) pseudequinum, $S$. (E.) latinum, $S$. (N.) cryophilum et $S$. (O.) nitidifrons. Les deux premières sont récoltées dans presque toutes les rivières; les autres, faiblement représentées quantitativement, se cantonnent dans un nombre de gîtes moins élevé. Quant à $S$. (N.) vernum, 
Tableau I.

Principales caractéristiques physiques des stations et abondance relative des différentes espèces.

$\mathbf{N}_{1}$ : numération des stations

$\mathrm{N}_{2}$ : numération des stations sur Boumaiza (1984)

$T_{m}$ : températures enregistrées durant les mois de novembre ou janvier

TM : températures enregistrées durant les mois de juin ou juillet

Substratum :
Nature du courant :

$$
\begin{aligned}
& \mathbf{m}=\text { moderé } \\
& \mathbf{r}=\text { rapide } \\
& \mathbf{l}=\text { lent }
\end{aligned}
$$

\begin{tabular}{|c|c|c|c|c|c|c|c|c|c|c|c|c|c|c|c|c|c|c|c|c|c|c|c|}
\hline $\mathbf{N}_{1}$ & $\mathbf{N}_{2}$ & STATIONS & $\begin{array}{l}\frac{E}{5} \\
\frac{5}{3} \\
\stackrel{5}{z} \\
z\end{array}$ & $\begin{array}{l}\frac{\dot{E}}{E} \\
\dot{E} \\
\dot{E} \underline{E} \\
\frac{E}{E}\end{array}$ & $\begin{array}{l}\dot{\dot{x}} \\
\underline{E} \\
\dot{\underline{g}}= \\
\underline{E}\end{array}$ & $\begin{array}{l}E \\
\frac{5}{5} \\
\frac{5}{5} \\
\frac{5}{5}\end{array}$ & $\begin{array}{l}5 \\
\pm \\
\pm \\
\vdots \\
5\end{array}$ & 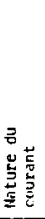 & 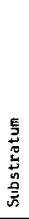 & 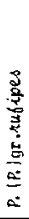 & 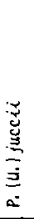 & 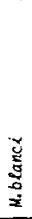 & 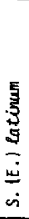 & 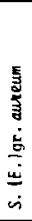 & $\begin{array}{l}\frac{5}{3} \\
\frac{3}{2} \\
\frac{2}{3} \\
5 \\
\frac{5}{3} \\
\dot{3}\end{array}$ & $\frac{1}{2}$ & 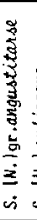 & $\begin{array}{l}8 \\
5 \\
5 \\
5 \\
5\end{array}$ & 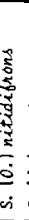 & 형 & 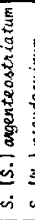 & 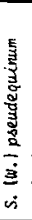 & অ \\
\hline 1 & (1) & Medjerda (Jendouba) & 146 & & $24^{\circ}$ & $>10$ & 11,16 & $m$ & $S$ & & & & & & & & & & & & & $\mathbf{1}$ & \\
\hline 2 & (2) & $" \quad$ (El Bathan) & 28 & $12^{\circ}$ & $24 \cdot 5$ & $>10$ & 0,16 & $\mathbf{r}$ & $\mathbf{p}$ & & & 1 & 3 & & & & & & & & & 4 & \\
\hline 3 & (14) & Mellegue I. cours > & 329 & $14^{\circ}$ & $26^{\circ}$ & $>10$ & 0,3 & $m$ & $\mathbf{S}$ & & & & 3 & & & & & & & & & 4 & \\
\hline 4 & $(15)$ & " II. cours moyen & 169 & $16^{\circ}$ & $26^{\circ}$ & $>10$ & 0,1 & $m$ & $\mathbf{P}$ & & & 3 & 2 & & & & & & & & & 4 & \\
\hline 5 & $(16)$ & " III, cours < & 138 & $16^{\circ}$ & & $>10$ & 0,1 & $\mathbf{r}$ & $\mathbf{S}$ & & & 1 & 4 & & & & & . & & & & 4 & \\
\hline 6 & $(12)$ & Tessa I. cours > & 295 & $16^{\circ}$ & & $>6$ & 0,3 & $m$ & $\mathbf{s}$ & & & 2 & 1 & & & & & & & & & 1 & \\
\hline 7 & (13) & "II. cours < & 132 & & & & 0,16 & $\mathbf{r}$ & 5 & & & & 2 & & & & & & & & & 1 & \\
\hline 8 & $(8)$ & Guezala (Fernana) & 230 & $8^{\circ}$ & $22^{\circ}$ & $<4$ & 0,4 & $\mathbf{r}$ & $\mathbf{P}$ & & & & 4 & & & & & & & & & 4 & \\
\hline 9 & $(7)$ & Bouheurtma & 133 & $14^{\circ}$ & $23^{\circ}$ & $>4$ & 0,1 & $m$ & $P$ & & & 1 & 4 & 2 & & & & & 1 & & & 4 & \\
\hline 10 & $(6)$ & Kesseb & 134 & $9^{\circ}$ & $23^{\circ}$ & $>6$ & 0,4 & $\mathbf{r}$ & $P$ & & & & 1 & & & & & & 1 & & & 3 & \\
\hline 11 & $(4)$ & Beja & 155 & & $31^{\circ}$ & $4-6$ & 0,5 & $r$ & $\mathbf{B}$ & & & 1 & 2 & & & & & & & & & 3 & \\
\hline 12 & $(3)$ & Zargua & 95 & $8^{\circ}$ & $23^{\circ}$ & $<5$ & 0,2 & $\mathbf{r}$ & $P$ & & & 1 & 2 & & 1 & & & & 1 & 1 & & 4 & \\
\hline 13 & $(11)$ & Khalled & 230 & $11^{\circ}$ & $19^{\circ}$ & $2-3$ & 1,3 & $\boldsymbol{T}$ & $\boldsymbol{p}$ & & & 4 & 4 & & & & & & 1 & & & 4 & \\
\hline 14 & $(9)$ & Silland I - Siliana & 420 & $16^{\circ}$ & $26^{\circ}$ & $<4$ & 1,5 & $\boldsymbol{m} \cdot \mathbf{r}$ & $\mathbf{P}$ & & & & 3 & & & & & & & & & 4 & \\
\hline 15 & & SIllana II - Lakouat & 375 & $18^{\circ}$ & & $<4$ & 0,5 & $\mathbf{r}$ & B & & & & 3 & & & & & & & & & 3 & \\
\hline 16 & & S1l Lana III.El Arroussa & 175 & $18^{\circ}$ & & $<4$ & 0,8 & $\mathbf{r}$ & G & & & 4 & 4 & & & & & & & & & 4 & \\
\hline 17 & $(10)$ & SIliana IV Testour & 78 & $14^{\circ}$ & $21^{\circ}$ & $<4$ & 0,3 & $\mathbf{r}$ & $\mathrm{C}$ & & & 4 & 4 & & 3 & & & & & & & 4 & \\
\hline 18 & $(16)$ & Emour & 400 & $11^{\circ}$ & $27^{\circ}$ & $<4$ & 4,5 & m & B & 1 & & & 4 & & 2 & & & & 4 & 1 & 1 & & \\
\hline 19 & $(19)$ & E1 Kebir (Tabarka) & $\theta$ & $16^{\circ}$ & $30^{\circ}$ & $>4$ & 0,1 & $\mathbf{r}$ & 5 & & & 1 & 3 & & & & & & 2 & 1 & 1 & 4 & \\
\hline 20 & $(20)$ & Renagha & 65 & & $26^{\circ}$ & $<4$ & 1 & $m$ & $P$ & & 1 & 1 & 4 & & 2 & & & & 3 & 1 & 1 & 4 & \\
\hline 21 & $(21)$ & Bouterfes & 23 & & $25^{\circ}$ & $>4 \mathrm{~T}$ & 0,7 & $\Gamma$ & $p$ & & & & 3 & & 1 & & & & 3 & & 1 & 4 & \\
\hline 22 & $(23)$ & El Madène & 30 & & & $>6$ & 0,4 & $\boldsymbol{T}$ & $\mathrm{P}$ & & & 1 & 3 & & & 1 & & & 1 & 1 & & 3 & 1 \\
\hline 23 & $(24)$ & Ziat ine & 6 & $14^{\circ}$ & $30^{\circ}$ & $>4$ & 0,25 & $m$ & 5 & & & & 1 & & & & & & & & & 1 & \\
\hline 24 & $(28)$ & Sedfenane & 100 & $12^{\circ}$ & $26^{\circ}$ & $>4$ & 0,08 & $\boldsymbol{T}$ & $P$ & & & & 2 & & & $\cdot$ & & & & 1 & & 3 & 1 \\
\hline 25 & $(27)$ & Melah (Dfalta) & 15 & $11^{\circ}$ & $26^{\circ}$ & $<4$ & 0,24 & $r$ & B & & & 3 & 3 & & & & & & & 1 & & 4 & 1 \\
\hline 26 & & Ghezala (Ichkeul) & 22 & & & $2-3 T$ & 0,5 & m & $P$ & & & & 1 & & & & & - & & & & 2 & \\
\hline 27 & $(25)$ & Joum Lne & 15 & $10^{\circ}$ & $28^{\circ}$ & $>4$ & 0,2 & $r$ & $P$ & & & & 3 & & & & & & & & & 2 & 1 \\
\hline 28 & $(30)$ & Mfliane I. (El Fahs) & 168 & $1^{0}$ & $26^{\circ}$ & $>4$ & 0,16 & m & $\mathrm{P}$ & & & & 3 & & & & & & & & & 4 & \\
\hline 29 & (31) & " II. (Mohammad Ia) & 34 & $5^{\circ}$ & $26^{\circ}$ & $>6$ & 0,15 & $\mathbf{r}$ & $P$ & & & & 3 & & & & & & & & & 4 & \\
\hline 30 & & El Kebir (Afft M1liane) & 192 & $13^{\circ}$ & $25^{\circ}$ & $3-4$ & 0,45 & $m$ & 5 & & & & 1 & & & & & & & & . & & \\
\hline 31 & (32) & Ab Id & 12 & $10^{\circ}$ & $29^{\circ}$ & $4-6$ & 0,18 & 1 & $\mathrm{~S}$ & & & & & & & & & & & & & 1 & \\
\hline 32 & $(36)$ & Nebhana & 22 & $9^{\circ}$ & & $4-6$ & 0,34 & $\mathbf{r}$ & $P$ & & & & 1 & & & & & & & & & 3 & \\
\hline 33 & (43) & Oasis Lella & 275 & $24^{\circ}$ & & $1-2$ & 0,77 & $m$ & $\mathrm{~S}$ & & & & & & & & & & & & & 4 & \\
\hline 34 & & Tamerza & 300 & & & $c 3$ & 1,5 & 1 & $\mathrm{~S}$ & & & & 3 & & & & & 4 & & & & 4 & \\
\hline 35 & $(45)$ & Das is Niefta & 75 & $26^{\circ}$ & & $1-3$ & 3,7 & m & $s$ & & & & & & & & & & & & & 1 & \\
\hline 36 & $(41)$ & El Harma & 52 & $12^{\circ}$ & & $<3$ & 0,2 & 1 & $\mathrm{~S}$ & & & & 1 & & & & 1 & 4 & & & & 1 & \\
\hline
\end{tabular}

Abondances relatives:

$$
\begin{aligned}
& 1=1 \text { à } 5 \text { individus } \\
& 2=5 \text { à } 10 \\
& 3=10 \text { a } 50 \\
& 4=>\text { à } 50 \text { individus. }
\end{aligned}
$$




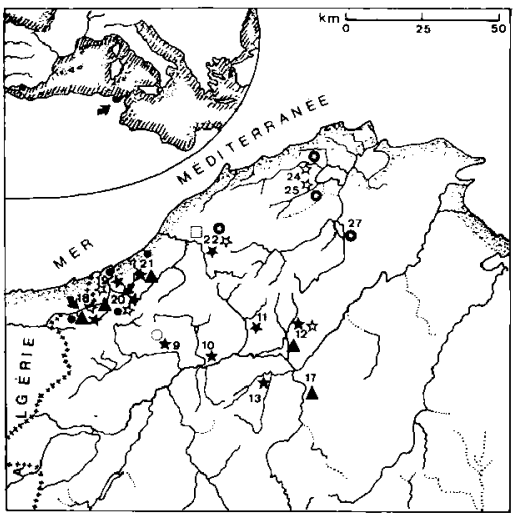

Fig. 2. Répartition de 9 espèces : flèche vers la droite : $P$. (P.) groupe rtefipes - flèche vers la gauche : $P$. (U.) juccii - triangles noirs: $S$. (N.) cryophilum - carré blanc: $S$. $(N$.) ibleum - étoiles noires: $S$. $(O)$ nitidifrons - étoiles blanches: $S$. (O.) ornatum - cercles noirs : $S$. (S.) argenteostriatum - points blancs cerclés noir : $S$. (W.) sergenti - cercle blanc : $S$. (E.) gr. aureum.

$S$. . (N.) yerburyi et $S$. (B.) ?erythrocephalum, leurs stations étant des ruisselets de montagne, ces taxa ne sont pas présents dans nos prèlèvements, sans doute à cause de leur situation plus élevée en altitude (600-900 m).

Six especes : $M$. blanci, S. (E.) gr. aureum, S. (N.) ruficome, S. (N.) gr. angustitarse, $S$. (O) omatum et $S$. (W.) sergenti sont nouvelles pour la Tunisie et quatre : $P$. $(P$.) gr. rufipes, $P$. (U.) juccii, $S$. (N.) ibleum et $S$. (S.) argenteostriatum, le sont pour l'Afrique du Nord.

A la station 14 (Oued Siliana), une larve mature présentait les caractéristiques du sous-genre Nevermannia : frontoclypeus, échancrure ventrale et processus tp de la mandibule ; par contre, l'histoblaste était différent de celui du sous-genre des régions paléarctiques : les quatre filaments très épais se terminaient par une extrémité effilée qui rappellent ceux de $S$. (N.) arabicum décrits par Crosskey (1982) d'Arabie Saoudite. D'autres prélèvements devraient nous informer sur la signification de cet aspect de l'histoblaste.

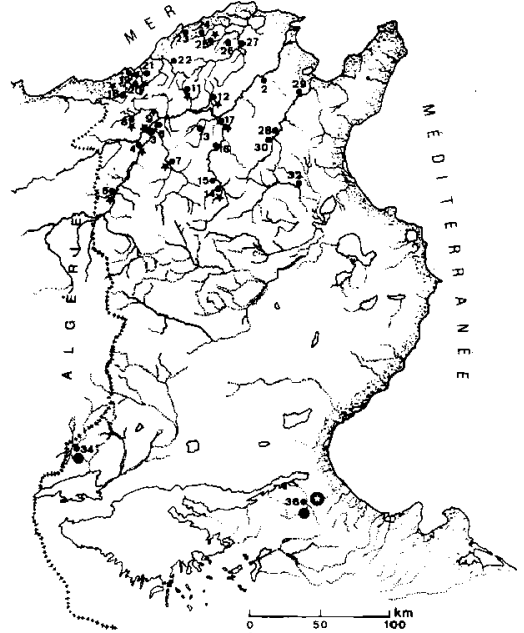

Fig. 3. Répartition de 4 espèces : petits cercles noirs: $S$. (E.) latinum - grands cercles noirs: $S$. (N.) ruficorne -étoiles noires : $M$. blanci - point blanc cerclé noir : $S$. (N.) angustitarse.

Sur les quatorze espèces recensées trois: $M$. blanci, $P$. (P.) gr. rufipes et $P$. (U.) juccii n'appartiennent pas au genre Simulium (Simulini) et se trouvent placées par Crosskey dans la tribu des Prosimulini

\section{Prosimulium (Prosimulium) gr. rufipes}

Commune dans toutes les régions montagneuses d'Eu rope centro-méridionale, l'espèce $\boldsymbol{P}$. $(\boldsymbol{P}$.) rufipes est présente jusqu'en Sicile. proche du territoire tunisien. Sa répartition altitudinale est très grande puisque Dorier (1962-1963) l'a trouvée en abondance entre 300 et $2000 \mathrm{~m}$ (la station la plus basse étant à $190 \mathrm{~m}$, La Drevenne, près de St Gervais). La larve est bien connue pour fréquenter les eaux à fort débit et bien oxygénées, au pied des cascades.

La nymphe du groupe rufipes a été capturée dans l'oued Ennour à $400 \mathrm{~m}$ d'altitude, au début du mois d'avril 1985. Cet affluent de l'oued Kébir prend naissance à $850 \mathrm{~m}$ d'altitude et se présente comme un torrent à fond de galets et de blocs, biotope 
habituel de l'espèce. La seule capture de la nymphe ne nous autorise pas à affirmer qu'il s'agit bien de l'espèce type; par rapport aux exemplaires alpins, il existe des différences très minimes dans l'arrangement des segments abdominaux et dans l'ordonnance des filaments branchiaux.

\section{Prosimulium (Urosimutium) juccii}

Découverte et décrite en Sardaigne par Contini (1966), l'espece n'était connue que dans cette île.

Elle a été retrouvée par nous dans l'oued Renagha, qui se situe dans une petite région au nord-ouest de la Tunisie, comprise entre la mer et les monts de Kroumirie : cette contrée se révèle particulièrement intéressante par la découverte de plusieurs taxons répandus dans l'ouest méditerranéen. L'exuvie nymphale, avec ses filaments respiratoires et sa tache si caractéristique à leur base, se trouvait dans un biotope correspondant exactement à celui decrit par Contini ; $P$. (U.) juccii supporte en effet une certaine eutrophie et des températures assez élevées $\left(15^{\circ}\right.$ en avril 1985 à $10 \mathrm{~h}) ;$ les eaux de l'oued sont riches en algues et contiennent des substances nutritives dérivant de la décomposition des végétaux (arbres et arbustes) assez denses qui bordent la rive. La découverte d'exuvies nymphales en avril tend à prouver que l'état larvaire se situe en hiver, comme en Sardaigne, et la nymphose en mars.

\section{Metacnephia blanci}

Décrite du Maroc (O. Rdat), l'espèce est signalée dans le Rif et le Haut-Atlas par Bailly-Choumara et Beaucournu-Saguez $(1978,1981)$. Sa limite septentrionale se situe en France méridionale, Sicile, Espagne et Balkans.

Capturée au nord de la Tunisie, $M$. blanci fréquente les ruisseaux ou les petites rivières à courant vif et eau bien oxygénée ; elle est cependant citée par BaillyChoumara et Beaucournu-Saguez dans des ruisseaux à courant faible à modéré.

Nettement moins eurytope que $S$. (W.) pseudequintm et $S$. (E.) latinum, son aire de distribution s'en trouve plus restreinte : d'une part, elle n'est jamais dominante dans les stations ; d'autre part, bien qu'elle tolère des salinités s'élevant jusqu'à $4 \%$, elle n'atteint pas les biotopes à température élevée situés au Sud.

\section{Simulium (Eusimulium) latinum}

De description relativement récente (Rubzov
1959-1964) (en Italie près de Salerme), appartenant au groupe aureum, l'espèce recouvre toute la région méditerranéenne occidentale (Italie, France du Sud, Espagne, Afrique du Nord...). La plupart du temps, elle est fréquente à basse altitude ; on l'observe en moins grande abondance dans le Rif et le Haut-Atlas jusqu'à $1500 \mathrm{~m}$ (Bailly-Choumara et BeaucournuSaguez 1978, 1981).

Elle était déjà signalée dans les ruisseaux nordtunisiens (Bailly-Choumara \& al. $1970-7$ gites sur 17) ; son aire de répartition (fig. 3) englobe la plus grande partie du territoire dans notre travail, jusque dans les oueds du sud tunisien. Son degré d'eurytopie est très élevé. Dans la plupart des gîtes où elle est présente, on remarque son abondance ; dans quelques stations de très basse altitude (St. 2 , $12,17 \ldots$..), $S$. (E.) latinum n'est représentée qu'en petit nombre, remplacée peu à peu par $S$. (W.) pseudequi. num. Les larves et nymphes sont observées le plus souvent sur les feuilles ou les herbes, et sur les pierres, dans les quelques stations dépourvues de végétation.

Espèces associées: toutes les espèces, avec une plus grande fréquence pour $S$. (W.) pseudequinum, puisque toutes les deux sont eurytopes.

\section{Simulium (Eusimulium) gr. aureum}

Beaucournu-Saguez (1977) avait déjà dénoncé la difficulté à séparer les espèces du groupe "aureum " en n'examinant que les stades immatures. Un certain nombre d'exuvies nymphales de ce groupe, différentes de l'espèce $S$. (E.) latinum, ont été capturées à la station 9 . Le filament supérieur des branchies nymphales est moins anguleux que l'espèce précédente; les deux inférieurs sont sessiles. La disposition de l'ensemble fait penser à l'espèce italienne $S$. (E.) paludicolum, mais en l'absence d'adultes, nous ne pouvons nous prononcer.

\section{Simulium (Nevermannia) cryophilum}

De très vaste répartition paléarctique, puisqu'il existe des gîtes connus depuis l'U.R.S.S. jusqu'en Angleterre. cette espèce se retrouve en Méditerra. née occidentale (Italie, Sicile, Sardaigne). Signalée pour la première fois en Afrique du Nord par Bailly. Choumara \& al. (1970), elle a été capturée par nous dans cinq stations $(12,17,18,20,21)$ de la même région; il s'agit là, sans doute, de sa limite la plus méridionale de distribution. D'après nos résultats, 
elle y semble très peu abondante; on la capture sur les fonds pierreux, dans des ruisseaux à courant vif, bien oxygénés et non pollués

Espèces associées : $S$. $(W$ ) pseudequinum $S$. (E.) latinum, $S$. (O.) nitidifrons, $M$. blanci.

\section{Simulium (Nevermannia) ibleum}

Décrite en Sicile par Rivosecchi (1966) comme une sous-espèce de S. (N.) angustitarse, elle a été élevée au rang d'espèce par Crosskey et Ashford (1981). Ce taxon a été signalé, au Liban et en Cyrénaique. Sa présence en Tunisie étend son aire de répartition à l'Afrique du Nord et sur le pourtour méditerranéen.

Comme la plupart des espèces du sous-genre Never. mannia, $S$. (N.) ibleum est commune dans les ruisselets de sources et dans les canaux d'irrigation; c'est une espèce du crénal et du rhithral. En Tunisie, elle est présente dans l'oued Madène, (St. 22) ruisselet à courant rapide, à fond pierreux avec végétation et à température fraîche. Comme au Liban, l'espèce sem. ble être représentée par une très faible population larvaire en avril.

Espèces associées: $S$. (E.) latinum, $S$. (O.) nitiditrons, $S$. (O.) ormatum et $S$. (W) pseudequinum.

\section{Simulium (Nevermannia) gr. angustitarse}

Dans le Sud (oued El Hamma), a été capturée une nymphe appartenant au groupe angustitarse.

Comme l'espèce type, le cocon est de structure très lâche et une très petite excroissance existe au milieu du bord antérieur. Il épouse la forme de la nymphe et se termine en pointe. Les filaments branchiaux ont tous les quatre le même diametre; ils ont sensiblement la même disposition que l'espèce type. La chétotaxie abdominale est également rigoureusement la même. Il faut signaler en outre, la présence de six longues et puissantes épines à la base des filaments branchiaux.

\section{Simulium (Nevermannia) ruficome}

Décrite à l'Ile de la Réunion, l'espèce est très répandue sur une vaste étendue de la région éthiopienne (Afrique intertropicale, du Sud, Madagascar, Iles Rodriguez et Maurice, Freeman et De Meillon, 1953) de la sous-région méditerranéenne (oasis présahariens: Roubaud, 1906; Algérie: Edwards, 1923 ; Maroc) des massifs montagneux isolés du Sahara tchadien (Tibesti, Tassili des Ajjer, Grenier \& Rioux 1960 . Grenier \& Clastier 1960) et au Moyen Orient (Crosskey 1967, Rivosecchi \& al. 1969).
Sa découverte dans le sud-tunisien établit la liaison entre les gîtes du Maroc - Sud de l'Espagne - Portugal, et ceux de la région éthiopienne. Larves et nymphes se trouvent situées dans deux petits cours d'eau méridionaux (oueds Tamerza et El Hamma) à faible débit et courant lent. Le fond est sableux, la végétation soit dense avec des roseaux et des algues (El Hamma), soit plus clairsemée avec des algues (Tamerza). L'espèce y est dominante sur les espèces associées : $S$. (E.) latinum et $S$. (W.) pseudequinum, encore bien représentées à Tamerza, mais peu fréquentes à El Hamma.

\section{Simulium (Odagmia) nitidifrons}

Décrite en Grande-Bretagne, l'espèce possède une vaste répartition géographique depuis l'Europe centrale et occidentale, jusqu'au Portugal (BeaucournuSaguez 1972) et à la bordure méditerranéenne : Maroc, où sa répartition altitudinale est aussi grande que dans les Pyrénées puisqu'elle monte jusqu'à $2000 \mathrm{~m}$ dans le Rif (Bailly-Choumara \& Beaucournu-Saguez 1978), Algérie : où Edwards l'avait notée dès 1923 près d'Alger et au Liban (Moubayed \& Clergue-Gazeau 1985).

En Tunisie, sa présence probable a été confirmée en 1970, dans le Nord du pays. Nos prospections ont permis de capturer $S$. (O.) nitidifrons dans une dizaine de gîtes situés au nord-ouest du territoire; si l'espèce semble présente en abondance au Maroc, elle n'est jamais dominante dans nos récoltes et atteint ici une de ses limites sud de distribution. Comme son biotope européen, elle est représentée dans les ruisseaux à courant rapide à moyen, bien oxygénés, à fond pierreux, pouvant comporter parfois des algues et des feuilles servant de support à la nymphe, et provenant d'une végétation bordante dense ou assez dense avec rives ombragées.

Espèces associées: $M$. blanci, $S$. (E.) latinum, S. (N.) cryophilum, S. (S.) argenteostriatum et $S$. (W.) pseudequinum.

\section{Simulium (Odagmia) ornatum}

De très large distribution paléarctique, l'espèce est commune surtout en plaine, moins fréquente lorsqu'on s'élève en altitude. S. (O.) omatum s'étend sur le territoire d'Afrique du Nord où elle est bien moins répandue que nitidifrons. Edwards (1923) l'avait trouvée en Algérie; elle a été observée au Maroc dans le Haut Rif jusqu'à $1550 \mathrm{~m}$. 
(Bailly-Choumara \& Beaucournu-Saguez 1978) et au Liban (Moubayed \& Clergue-Gazeau 1985). Jusqu'à présent, elle était inconnue en Tunisie. Dans nos prélèvements, sa densité est très faible et sa présence limitée aux stations situées à l'extrême Nord du pays. Dans celles-ci, le biotope se caractérise par un courant rapide, bien oxygéné. Nymphes et larves sont souvent capturées sur les végétaux.

\section{Simulium (Simulium) argenteostriatum}

Décrite par Strobl en 1898 , l'espèce a été redécrite indépendamment par Séguy \& Dorier (1936) sous le nom de S. rupicolum; les deux noms ont été mis en synonymie par Zwick (1974). C'est une espèce de montagne; son aire de distribution comprend les Alpes, les Pyrénées et les Apennins ; en altitude, on la trouve entre 170 et $1700 \mathrm{~m}$. Fréquentes entre 300 et $1300 \mathrm{~m}$, larves et nymphes sont adaptées aux courants les plus forts.

Les larves, nymphes et exuvies nymphales capturées sont semblables à celles de l'espèce type, les larves avec cependant un frontoclypeus clair. Cette espèce a été retrouvée dans quatre ruisseaux rapides du nord-ouest (st. 18, 19, 20, 21), proches l'un de l'autre.

\section{Simulium (Wilhelmia) pseudequinum}

L'espèce est d'origine orientale; sa répartition couvre une aire continue: nord-ouest de l'Inde, URSS méridionale, Balkans et tout le pourtour méditerranéen.

Mentionnée depuis longtemps par Colas-Belcour (1931) dans un ruisseau à température élevée à Tozeur, décrite indépendamment par Puri (1925 et 1933), elle a été retrouvée en 1970 dans la majorité des gites prospectés par Bailly-Choumara. De loin la plus abondante, elle englobe tous les réseaux hydrographiques du Nord au Sud du pays (fig. 1), et conquiert tous les types de biotopes, même ceux qui ont une faible pollution organique. Les substrats pierreux sont favorables à l'espèce, elle devient plus rare dans ceux à fonds sableux et disparait sur les fonds vaseux.

\section{Simulium (Wilhelmia) sergenti}

Décrite sur du matériel algérien (oued Ksour, près de Biskra) et retrouvée jusqu'à la limite méridionale des hauts plateaux, l'espèce a été capturée dans de nombreux gîtes au Maroc depuis le Rif au Nord jusqu'aux oueds des oasis présahariens du Sud. Sa limite septentrionale se situe au Portugal (Beaucournu-Saguez 1975) et au sud-est de l'Espagne (Beaucournu-Saguez 1972).

Ce taxon n'avait pas encore été observé en Tunisie; il est présent dans quatre cours d'eau proches l'un de l'autre (fig. 2), tributaires du lac Ichkeul ; en nombre restreint contrairement aux gites marocains et algériens, les larves et les nymphes se trouvaient au milieu de celles de $S$. (W.) pseudequinum et $S:(E$. latinum largement dominantes. Dans ses notes de 1972 et 1975, Beaucournu-Saguez indique une certaine variété de biotopes de l'espèce; il n'en est pas de même dans le cas présent où il s'agit exclusivement de biotopes à végétation bordante rare, avec rives parfois complètement dénudées ; le courant est rapide, le fond parsemé de galets.

\section{4. - Discussion et conclusion}

18 taxons sont actuellement recensés en Tunisie : 14 cités dans cette note, auxquels s'ajoutent 4 espèces signalées par Bailly-Choumara et al. (1970). La présence de $S$. (S.) reptans reste incertaine.

Deux espèces, $S$. (W.) pseudequinum et $S$. (E.) latinum sont les plus abondantes. Toutes deux, par leur densité, s'opposent aux espèces des biotopes du nord-ouest que l'on trouve en plus faible quantité.

Sur les 18 espèces répertoriées dans ce travail et dans celui de Bailly-Choumara \& al (l.c.), 15 sont localisées dans la région située entre la mer Méditerranée et les monts de Kroumirie. Cette grande richesse spécifique, observée dans les bassins versants du nord-ouest, diminue au fur et à mesure que l'on se dirige vers les ruisseaux de la partie sud du pays, où les eaux présentent une salinité beaucoup plus élevée (Boumaiza 1984) et d'importantes amplitudes thermiques. L'espèce ubiquiste $S$. (W.) pseudequinum est la seule présente dans les eaux thermales des oasis: Tozeur, Lella et Nefta.

Ce premier inventaire faunistique a amélioré nos connaissances en ce qui concerne les espèces des cours moyens et inférieurs; l'amplitucie altitudinale étant faible (de l'ordre de $400 \mathrm{~m}$ ). l'examen de prélèvements situés à des altitudes plus élevées devrait nous permettre d'une part, de déterminer de nouvelles espèces et, d'autre part, de retrouver celles inventoriées par Bailly-Choumara et al. (1970), dans les montagnes de Kroumirie. 
L'analyse biogéographique du peuplement montre qu'il s'agit d'une faune paléarctique largement répartie en Europe et dans la sous-région méditerranéenne. Deux espèces seulement sont connues de régions avoisinantes:

1) $S$. (N.) ruficome, d'origine éthiopienne; repérée dans deux cours d'eau méridionaux (oued Tamerza et oued El Hamma), elle y supplante les espèces associées : $S$. (E.) latinum et $S$. (W.) pseudequinum;

2) $S$. (W.) pseudequinum, d'origine orientale, omniprésente dans les différents cours d'eau prospectés.

Parmi les éléments paléarctiques, plusieurs groupes peuvent être distingués :

- un premier, avec deux especes alpines, dont l'aire recouvre les régions montagneuses d'Europe centrale et méridionale; $P$. (P.) gr. rufipes et $S$. (S.) argenteostriatum. Leur présence dans le nord-ouest tunisien mérite d'ètre soulignée : il n'est pas exclu qu'elles puissent se retrouver sur les hauteurs des plateaux est-algériens non encore prospectés ;

- un deuxième groupe se compose d'éléments à distribution ouest-méditerranéenne : $M$. blanci, $S$. (E.) latinum, $S$. (N.) cryophilum et $S$. (W.) sergenti.

- le dernier groupe comprend les espèces circum-méditerranéennes : $S$. (N.) ibleum, $S$. (N.) nitidifrons et $S$. $(O$.) ornatum.

La présence d'une espèce endémique de Sardajgne, $P$. (U.) juccii, en Tunisie mérite une mention particulière. Fait-elle partie de cette faune spécialisée du vaste continent tyrrhénéen qui existait au montien (paléogène)? Ce continent s'est effondré au cours de l'éocène moyen et il n'en reste aujourd'hui que des vestiges tels que : la Sardaigne, la Corse, quelques îles méditerranéennes, le nord-est algérien et le nord-ouest tunisien ; cette hypothèse expliquerait la présence de l'espèce en Kroumirie et son absence sur le continent européen. C'est le cas également pour d'autres espèces (appartenant à d'autres groupes et notamment à celui des Coléoptères), isolées dans certaines îles méditerranéennes et qui se rencontrent dans cette région tunisienne.

Un certain nombre d'espèces communes existent entre le peuplement simulidien du nord-ouest de la Tunisie et celui des régions voisines : 13 espèces avec l'Italie et 8 avec l'ouest du Maghreb. La pénétration de $S$. (W.) sergenti et de $S$. (N.) ruficome à partir de l'Afrique du Nord jusqu'au sud de la péninsule ibérique (et sans atteindre l'Italie), s'est vraisem- blablement effectuée à partir du montien. Ces deux contrées ont en effet connu des phases de communication entre elles avant l'ouverture du détroit de Gilbraltar.

Travaux cités

Anderson (C.) \& Lehucher (P.). 1940. - Premier cas d'Onchocercose cutanée observée en Tunisie. Arch. Inst. Pasteur ; Tunis., 29 (f) : 105- 112.

Baitly.Choumara (H.) \& Beaucournu-Saguez (F.). 1978. Contribution à l'étude des Simuliess du Maroc (Diptera. Simuliidae) 1. Le Rif. Bull. Inst. Scient., Rabat., (5) : 121-144.

Bailly-Choumara (H.) \& Beaucournu-Saguez (F.). 1981. Contribution à l'étude des Simulies (Diptera. Simuliidae) du Maroc 2) le Haut-Atlas. Bull. Inst. Scient, Rabat, (5) : 39.57.

Bailly-Choumara (H.), Bernard (M.R.), Grenier (P.), Le Roy-Moret (M.C.) \& Mouchet (J.) 1970. - Note fauristique sur les Simulies (Diptera. Simuliidae) du Nord de la Tunisie. Cah. O.R.S.T.O.M., sér. Ent. Méd. Parasitol., 8 (4) : 377-382.

Beaucournu-Saguez (F.). 1972. - Captures de Simulies dans la moitié Sud du Portugal. Premières captures en Europe de Simulium sergenti Edwards 1923 et de Simulium ruficome Macquart 1838. An. Esc. nac. Saude publ. Med. trop. 6: 73.83.

Beaucournu-Saguez (F.). 1975. -- Récoltes de Simulies (Diptera, Simuliidae) dans le Sud-Est de l'Espagne. Ann. Soc. Ent. Fr., (N.S.), 11 (1): 73.89 .

Beaucournu-Saguez (F.). 1977. - Contribution à l'étude de Simulies (Diptera. Nematocera) du complexe * aureum * en France. Ann Parasit., 52 (2) : 181-194.

Boumaiza (M.). 1984. - Contribution à la limnologie de la Tunisie. Etude physico-chimique. Archs. Inst. Pasteur, Tunis., 61 (2-3) : 205-246.

Clastrier (J.) Grenier (P.). 1961 - Nouvelles récoltes de Simulium nuficome Macq. au Sahara. Arch. Inst. Pasteur Algérie, 39 : 106-107.

Colas-Belcour (J.). 1931. -- Note sur la faune parasitologique des oasis de Tozeur et Kébili. Arch. Inst. Pasteur, Tunis., 20 (I): 66-72.

Contini (C.). 1966 . - * Urosimulium jucci n. sp. (Diptera, Simuliidae) nuova specie di Simulide della Sardegna * Riv. Parass., $27: 269-289$.

Crosskey (R.W.). 1967. - A preliminary revision of the black-flies (Diptera. Simuliidae) of the Middle East. Trans. R. Ent. Soc. Lond. $119: 1-45$.

Crosskey (R.W.). 1981. - Simuliid Taxonomy The contemporary Scene : 3-18. In * Blackflies, the future for biological merhods in integrated control: Laird M. ed. Academic Press. London 399 p.

Crosskey (R.W.) \& Ashford (R.W.). 1981. - The occurrence of Simulium S. I. in the libyan Arab Republik. Ann. Trop. Med. Parastiol. 75 (6) : 647-651.

Crosskey (R.W.) \& Buttiker (W.). 1982. - Insects of Saudi Arobia. Diptera: Fam. Simuliidae. Fauna of Saudi Arabia : 398-446.

Dorier (A.). 1962-1963. - Documents pour servir à la connaissance des Simuliidae de Sud-Est de la France. Trav. Labor. Hydrob. Pisc. Uniw. Grenoble 54-56: 7-79.

Edwards (F.W.). 1923. - On some algerian species of Simulium. Arch. Inst. Pasteur, Algerie 1 : 647-653.

Freeman (P.) \& De Meillon (B.). 1953. - Simuliidae of the Ethio pian Region. British Museum (Natural History). London VI + $224 \mathrm{p}$.

Grenier (P.). 1953. - Simuliidae de France et d Afrique du Nord. Encycl. Ent. sèr. A. $29: 1-170$.

Grenier (P.) \& Clastrier (J.). 1960. - Une Simulie saharienne : Simu lium ruficome Macquart Arch Inst. Pasteur, Algénie 38 : 329-330. 
Grenier (P.), Faure (P.R.) \& Laurent (J.). 1957. - Simulies (Diptera, Simuliidae) du Maroc (deuxième mémoire). Arch. Inst. Pasteur Maroc $5(6)$ : 218-242.

Grenier (P.) \& Rioux (J.A.). 1960. - Présence de Simulium ruficome Macquart, 1838 (Diptera, Sirnuliidae) au Tibesti. Mission épidémiologique au Nord Tchad. Centre d'information et d'études des problèmes humains dans les zones arides 106:3 p.

Grenier (P.) \& Theodorides (J.). 1953. - Simulies (Dipt. Simuliidae) du Maroc. Arch. Inst. Pasteur, Maroc 4 (6) : 429-441.

Moubayed (Z.) \& Clergue-Gazeau (M.). 1985. - Les Simuliidae (Diptera) de trois rivières (Oronte, Litani et Beyrouth) du Liban. Annls. Limnol. 21 (1): 83-88.

Puri (I.M.). 1925. - A note on the early stages of a species of Simu. lium from Tunis, S. equinum var, mediterraneum, nov. (Diptera, Simuliidae). Ann. Mag. Nat. Hist. 16: 253-255.

Puri (I.M.). 1933. - Studies on Indian simuliidae. Part IX. S. equinum var. mediterraneum Puri and S. paraequinum sp. n. Ind. Joum. Med. Res. 21 (1): 11:16.
Rivosecchi (L.). 1966. - Contributo alla conoscenza dei simulidi italiani. XIV: sul gruppo angustitarse. Riv. Parass. 27 (3) : 185-202.

Rivosecchi (L.). 1978. - Gtide per il riconoscimento delle specie animali delle acque interne italiane. 3. Simuliti (Diptera, Simuliidae). Consiglio Nationale delle Ricerche: $88 \mathrm{p}$.

Rivosecchi (L.). 1978. - Simuliidae Diptera Nematocera. Fauna Italia, 13. VIII. 533 p. Accademia Nazionale Ital ia di Entomol. e dell Unione Zool. Italiana. Bologna.

Roubaud (M.E.). 1906. - Aperçus nouveaux morphologiques et biologiques sur tes Diptères piqueurs du groupe des Simulies, C.R. Acad. Sc. Paris 143: 519-521.

Sézuy (E.). 1921. - Faune entomologique des Iles Canaries. Séjour de M.P. Lesne dans la Grande Canarie (1902-1903). II Dipteres piqueurs, Bull. Mus. Nat. Hist. Nat. Paris 17; 291-295.

Séguy (E.) 1925. - Description d'un notveau Simulitum et synopsis des espèces méditerranéennes (Dipt. Simul.), Eos 1:231-238. 